\title{
Bone marrow examination in patients with Ewing sarcoma/peripheral primitive neuroectodermal tumor without metastasis based on ${ }^{18} \mathrm{~F}$-fluorodeoxyglucose positron emission tomography/computed tomography
}

\author{
Chiaki Inagaki ${ }^{1,2}$. Tatsunori Shimoi ${ }^{1} \cdot$ Hitomi Sumiyoshi Okuma ${ }^{1} \cdot$ Asuka Kawachi $^{1} \cdot$ Kazuki Sudo $^{1}$. \\ Akihiko Shimomura ${ }^{1}$ - Emi Noguchi ${ }^{1}$ - Makoto Kodaira ${ }^{1}$ - Mayu Yunokawa ${ }^{1} \cdot K$ Kan Yonemori ${ }^{1}$. Chikako Shimizu ${ }^{1}$. \\ Ayumu Arakawa $^{3} \cdot$ Chitose Ogawa $^{3} \cdot$ Akihiko Yoshida $^{4} \cdot$ Yasuhiro Fujiwara ${ }^{1} \cdot$ Kenji Tamura $^{1}$
}

Received: 26 March 2019 / Accepted: 26 April 2019 / Published online: 18 May 2019

(c) The Author(s) 2019

\begin{abstract}
Ewing sarcoma/peripheral primitive neuroectodermal tumor (ES/PNET) is an aggressive bone tumor. Bone marrow aspiration and biopsy (BMAB) has been recognized as the gold standard for assessing bone marrow status. While the latest guideline suggests the need to omit bone marrow aspiration in patients with no findings on ${ }^{18} \mathrm{~F}$-fluorodeoxyglucose positron emission tomography $\left({ }^{18} \mathrm{~F}\right.$-FDG PET) based on one retrospective report, there is no study using ${ }^{18} \mathrm{~F}$-FDG PET/computed tomography $(\mathrm{CT})$. We retrospectively reviewed 26 consecutive, previously untreated, ES/PNET patients. We compare the results of bone marrow aspiration and biopsy (BMAB) and those of ${ }^{18} \mathrm{~F}$-FDG PET/CT in ES/PNET patients. All of the 21 patients without metastases on ${ }^{18} \mathrm{~F}-\mathrm{FDG}$ PET/CT had negative BMAB. The sensitivity of bone marrow involvement in bone metastases positive patients on ${ }^{18} \mathrm{~F}$-FDG PET/CT was $75 \%(3 / 4)$, and the specificity was $100 \%(22 / 22)$. In addition to the metastatic findings on ${ }^{18} \mathrm{~F}$-FDG PET/CT, tumor diameter, lactate dehydrogenase level at diagnosis, and the presence or absence of bone metastasis were factors related to bone marrow involvement. It may be a reasonable option to omit BMAB in ES/PNET patients with no distant metastasis based on ${ }^{18} \mathrm{~F}-\mathrm{FDG}$ PET/CT findings.
\end{abstract}

Keywords Ewing sarcoma/peripheral primitive neuroectodermal tumors $\cdot{ }^{18} \mathrm{~F}$-FDG PET/CT $\cdot$ Bone marrow involvement

Electronic supplementary material The online version of this article (https://doi.org/10.1007/s12032-019-1279-8) contains supplementary material, which is available to authorized users.

Tatsunori Shimoi

tshimoi@ncc.go.jp

1 Department of Breast and Medical Oncology, National Cancer Center Hospital, Tokyo 104-0045, Japan

2 Department of Frontier Science for Cancer and Chemotherapy, Osaka University Graduate School of Medicine, Osaka, Japan

3 Department of Pediatric Oncology, National Cancer Center Hospital, Tokyo, Japan

4 Department of Pathology and Clinical Laboratories, National Cancer Center Hospital, Tokyo, Japan

\section{Introduction}

Ewing sarcoma/peripheral primitive neuroectodermal tumor (ES/PNET) is a member of the Ewing sarcoma family of tumors and originated from small undifferentiated neuroectodermal cells. ES/PNET is histologically characterized by small blue round cells and molecularly defined by the fusion of FET (FUS, Fused in Sarcoma; EWS, Ewing sarcoma breakpoint region 1, [also abbreviated EWSR1]; and TAF15, TATA box binding protein associated factor $68 \mathrm{kDa}$ ) gene family with ETS (E26 transformation-specific) family; with the most common fusion being EWSR-1-FLT1 (Fmsrelated tyrosine kinase 1) [1, 2]. ES/PNET mainly affects adolescents and young adults, and has an aggressive nature.

Metastatic status at diagnosis is the most significant prognostic factor of ES/PNET [3]. Patients with localized disease can expect $70-80 \%$ of 5-year overall survival. On the other hand, patients with metastatic disease, who 
accounts for approximately $15-20 \%$ of newly diagnosed patients, have a significantly worse 5-year overall survival of less than 30\% [4]. The most common sites of metastases are the lung, bone, and bone marrow. Patients with isolated lung metastases show better survival compared to those with bone metastases, or to those with a combination of lung and bone metastases [3]. Moreover, some studies revealed that bone marrow involvement is an independent unfavorable prognostic factor [5-8]. Therefore, staging of ES/PNET must be oriented toward the detection of the lung, bone, and bone marrow metastases.

Several guidelines recommend an extensive workup, including chest computed tomography (CT), magnetic resonance imaging (MRI) of the primary site, bone scan, ${ }^{18} \mathrm{~F}$-fluorodeoxyglucose positron emission tomography/ CT $\left({ }^{18} \mathrm{~F}\right.$ - FDG PET/CT), and bone marrow aspiration and biopsy (BMAB). However, these are expert opinionbased, and optimal combination of imaging modalities is not fully determined $[9,10]$. A bone scan is a conventional method used for the detection of bone metastasis. Recently published literature also reported that ${ }^{18} \mathrm{~F}$-FDG PET/CT and ${ }^{18}$ F-FDG PET possess high sensitivity and specificity in detecting bone metastases compared to bone scan and MRI [11]. For the evaluation of bone marrow involvement, BMAB is recognized as the gold standard technique, and MRI of the spine and pelvis as an alternative. Bilateral $\mathrm{BMAB}$ seems to be superior to unilateral BMAB because a previous retrospective report showed the discrepancies between bilateral samples; however, the necessity of bilateral BMAB in the initial staging is not well defined [12]. Moreover, evidence of a bone marrow involvement does not affect the treatment strategy in the presence of distant metastases. Furthermore, European Society of Medical Oncology Clinical Practice Guidelines on bone sarcomas stated that it will be possible to omit bone marrow aspiration when there is no evidence of metastatic disease on ${ }^{18}$ F-FDG PET scan [9]. This statement was based on the only one retrospective study report, which showed that bone marrow involvement found on BMAB is closely associated with the detection of metastatic osseous disease by ${ }^{18}$ F-FDG PET and bone scan $[9,13] .{ }^{18} \mathrm{~F}$-FDG PET/CT provides more accurate anatomical localization of metabolic abnormalities compared to ${ }^{18} \mathrm{~F}$-FDG PET. Therefore, recently, ${ }^{18} \mathrm{~F}$-FDG PET/CT is becoming more widely used in daily practice for the initial staging of ES/PNET [14]. However, there has been no study examining the association between bone marrow involvement and ${ }^{18} \mathrm{~F}$-FDG PET/ CT findings.

In this study, we retrospectively compared the concordance in the metastatic status of ES/PNET between the results of BMAB and ${ }^{18} \mathrm{~F}-\mathrm{FDG}$ PET/CT. We aimed to confirm that bone metastases on ${ }^{18} \mathrm{~F}$-FDG PET/CT accurately indicated bone marrow metastasis on $\mathrm{BMAB}$.

\section{Materials and methods}

\section{Patients}

We retrospectively reviewed consecutive, previously untreated, ES/PNET patients who were older than 10 years and were diagnosed at the National Cancer Center Hospital $(\mathrm{NCCH})$ between January 1, 2010, and December 31, 2016. All patients were histologically confirmed as ES/PNET with biopsy specimens. We included patients who underwent ${ }^{18} \mathrm{~F}$ FDG PET/CT and BMAB of the iliac crest at the initial assessment. We excluded all patients with an incomplete medical record. We performed a comprehensive review of each patient's medical history, including patients' characteristics, laboratory tests, pathological reports, and imaging diagnosis, to identify the localized or metastatic disease. We categorized primary sites into extremities, chest, spine, pelvis, and others. We also measured the largest primary tumor diameter. We compared the concordance rate in metastatic status between the results of BMAB and ${ }^{18} \mathrm{~F}$-FDG PET/CT.

\section{Ethics approval and consent to participate}

The NCCH Institutional Review Board approved this study (No. 2012-335). Because this was a retrospective study, written informed consent was not obtained from the subjects. At $\mathrm{NCCH}$, for retrospective clinical studies such as case treatment effects, we confirm with patients the consent for comprehensive clinical studies. All participants or parents (for below 16 yrs of age) in this study agree on its comprehensive research participation.

\section{${ }^{18}$ F-FDG PET/CT imaging}

All patients underwent ${ }^{18} \mathrm{~F}$-FDG PET/CT before the first dose of the treatment. Blood glucose level was not sampled at the time of FDG injection because all patients were nondiabetic. Independent specialized radiologists assessed all ${ }^{18} \mathrm{~F}-\mathrm{FDG}$ PET/CT imaging to determine whether any bone lesion was observed or not. We determined the metastatic status and obtained the sites of metastases according to the radiologist's statement on the imaging reports.

\section{Bone marrow sampling}

We sampled all BMAB from the iliac crest unilaterally before the commencement of treatment. When primary tumors involved a portion of the ilium, we exclusively performed $\mathrm{BMAB}$ contralateral to the primary tumor site to prevent contamination. For each patient, we assessed the bone marrow involvement status by the BMAB pathological 
report. We determined bone marrow involvement to be positive if the pathologist's statement on either the bone marrow aspiration (BMA) or bone marrow biopsy (BMB) was positive.

\section{Statistical analysis}

Clinical data of the patients were extracted from the medical records. For the comparison of continuous variables between the two groups, the Wilcoxon rank-sum test was used for the statistical analysis. For the comparison of proportions between the two groups, Fisher's exact test was used. We used JMP software, version 11 (SAS Institute Japan, Cary, CA, USA) for all statistical analyses. Values of $p<0.05$ indicated statistically significant differences.

\section{Results}

\section{Patient characteristics}

Of the 36 patients diagnosed with ES/PNET during the study period, 26 were included in the analysis. Ten patients were excluded due to incomplete initial work-ups. Patients and disease characteristics are listed in Table 1. All patients had good Eastern Cooperative Oncology Group performance status (ECOG PS) of zero, at initial diagnosis. The median age was 26.3 (range 11-53) years, and more than half of the patients were male $(n=14)$. All except one patient underwent combination imaging, which consisted of ${ }^{18} \mathrm{~F}-\mathrm{FDG}$ PET/CT $(n=26)$, contrasted CT $(n=25)$, and MRI of the primary site $(n=11)$, at the initial assessment.

Based on ${ }^{18} \mathrm{~F}-\mathrm{FDG}$ PET/CT, 21 patients had localized disease, and five patients had metastatic disease (Table 1). None of the patients had discordance in metastatic status between imaging modalities $\left({ }^{8} \mathrm{~F}-\mathrm{FDG}\right.$ PET/CT, contrasted $\mathrm{CT}$, and MRI). Of patients with metastasis, the most common site was the bone (four patients), followed by the lung (three patients), the lymph nodes (two patients), the liver (one patient), and the breast (one patient).

When we compared patients with localized and metastatic disease, primary tumor size $\geq 80 \mathrm{~mm}(p=0.0038)$ and serum lactate dehydrogenase (LDH) level (greater than twice the upper limit of normal) $(p=0.0038)$ were significantly higher in metastatic patients (Supplementary Table 1).

\section{The concordance between bone marrow samples and ${ }^{18} \mathrm{~F}-\mathrm{FDG} \mathrm{PET} / \mathrm{CT}$ results}

Three patients had positive BMAB, while 23 patients had negative BMAB (Table 2). All of the 21 patients with localized disease on ${ }^{18} \mathrm{~F}$-FDG PET/CT had negative BMAB. All three patients with positive BMAB showed
Table 1 Study population

\begin{tabular}{|c|c|}
\hline Characteristics & $\begin{array}{l}\text { Total }(\%) \\
n=26\end{array}$ \\
\hline \multicolumn{2}{|l|}{ Age at diagnosis (years) } \\
\hline$\leq 20$ & $10(38)$ \\
\hline $21-30$ & $8(31)$ \\
\hline $31-40$ & $5(19)$ \\
\hline 41 & $3(12)$ \\
\hline \multicolumn{2}{|l|}{ Gender } \\
\hline Male & $14(54)$ \\
\hline Female & $12(46)$ \\
\hline \multicolumn{2}{|l|}{ Primary tumor site } \\
\hline Extremities & $3(12)$ \\
\hline Chest wall & $4(15)$ \\
\hline Spine & $2(8)$ \\
\hline Pelvis & $7(27)$ \\
\hline Others & $10(38)$ \\
\hline \multicolumn{2}{|l|}{ Metastatic status } \\
\hline No metastasis (localized) & $21(81)$ \\
\hline Metastatic & $5(19)$ \\
\hline \multicolumn{2}{|l|}{ Metastatic site } \\
\hline Bone & $4(15)$ \\
\hline Lung & $3(12)$ \\
\hline Lymph node & $2(8)$ \\
\hline Liver & $1(4)$ \\
\hline Breast & $1(4)$ \\
\hline \multicolumn{2}{|l|}{ Primary tumor size } \\
\hline Median (range) (mm) & $72(10,176)$ \\
\hline$\geq 80 \mathrm{~mm}(n, \%)$ & $10(38.5)$ \\
\hline \multicolumn{2}{|l|}{ Serum LDH level } \\
\hline Median (range) (mg/dL) & $179.5(105,5183)$ \\
\hline$\geqq 2 \times \operatorname{ULN}(\mathrm{n}, \%)$ & $3(11.5)$ \\
\hline
\end{tabular}

LDH; lactate dehydrogenase, ULN; the upper limit of normal

bone metastasis on ${ }^{18} \mathrm{~F}$-FDG PET/CT. The sensitivity of bone marrow involvement in bone metastases positive patients on ${ }^{18} \mathrm{~F}$-FDG PET/CT was $75 \%$ (3/4), and the specificity was $100 \%$ (22/22). More specifically, of the three patients with positive BMAB that showed bone metastasis on ${ }^{18}$ F-FDG PET/CT, two had multiple osseous lesions (one had metastases to the vertebrae, pelvis, and femur while the other had metastases to the skull, clavicle, scapula, sternum, rib, humerus, vertebrae, femur, tibia, and fibula); the third patient had solitary osseous lesion (vertebra) $(p=0.0015)$. Moreover, of those with negative BMAB results, one patient with bone metastases had multiple osseous lesions (sternum, vertebrae, and pelvis). Of the patients with metastatic disease on ${ }^{18} \mathrm{~F}$-FDG PET/CT, $60 \%$ $(3 / 5)$ had a positive BMAB. Primary tumor size $\geq 80 \mathrm{~mm}$ $(p$ value $=0.046)$ and serum LDH level (greater than twice the upper limit of normal) $(p$ value $=0.0004)$ were 
Table 2 The result of bone marrow metastatic status and patients' characteristics

\begin{tabular}{|c|c|c|c|}
\hline & $\begin{array}{l}\text { BM met positive } \\
(n=3)\end{array}$ & $\begin{array}{l}\text { BM met negative } \\
(n=23)\end{array}$ & $p$ value \\
\hline Gender (male) & & & 0.63 \\
\hline Male & 2 & 12 & \\
\hline Female & 1 & 11 & \\
\hline Age at diagnosis & & & 0.96 \\
\hline Median (range) & $27.5(17,38)$ & $32(11,53)$ & \\
\hline Primary tumor site & & & 0.41 \\
\hline Pelvis & 2 & 5 & \\
\hline Non-pelvis & 1 & 18 & \\
\hline Primary tumor size & & & $0.046^{*}$ \\
\hline$\geq 80 \mathrm{~mm}(n, \%)$ & $3(100)$ & $7(30.4)$ & \\
\hline Serum LDH level & & & $0.0004 *$ \\
\hline$\geqq 2 \times \operatorname{ULN}(\mathrm{n}, \%)$ & $3(100)$ & $0(0)$ & \\
\hline $\begin{array}{l}\text { Distant metastases } \\
\text { on }{ }^{18} \text { F-FDG PET/ } \\
\text { CT }\end{array}$ & & & $0.0038^{*}$ \\
\hline Positive & 3 & 2 & \\
\hline Negative & 0 & 21 & \\
\hline Bone metastases & & & $0.0015^{*}$ \\
\hline $\begin{array}{l}\text { Yes (multiple/ } \\
\text { solitary) }\end{array}$ & $3(2 / 1)$ & $1(1 / 0)$ & \\
\hline No & 0 & 22 & \\
\hline Lung metastases & & & 0.32 \\
\hline Yes & 1 & 2 & \\
\hline No & 2 & 21 & \\
\hline
\end{tabular}

$B M$ met bone marrow metastasis, $L D H$ lactate dehydrogenase, $U L N$ the upper limit of normal, ${ }^{18} \mathrm{~F}$-FDG PET/CT ${ }^{18} \mathrm{~F}$-fluorodeoxyglucose positron emission tomography/computed tomography

*Statistically significant

significantly higher in BMAB-positive patients compared to negative patients.

\section{Discussion}

In this study, we retrospectively assessed the utility of ${ }^{18} \mathrm{~F}$ FDG PET/CT in the initial assessment to predict ES/PNET in adolescent and young adult patients with ES/PNET who also had bone marrow involvement. We revealed a high sensitivity $(75 \%)$ and specificity $(100 \%)$ of ${ }^{18} \mathrm{~F}-\mathrm{FDG}$ PET/CT in the assessment of bone marrow involvement. The result of this study indicated that having no metastatic site following ${ }^{18} \mathrm{~F}-\mathrm{FDG}$ PET/CT is an accurate negative indicator of bone marrow involvement. More specifically, all three patients with bone marrow involvement had bone metastatic on ${ }^{18} \mathrm{~F}$ FDG PET/CT.

Bone marrow involvement is a common event in metastatic ES/PNET and is considered an independent negative prognostic factor among ES/PNET patients with metastases $[5-8,15]$. Therefore, the identification of bone marrow involvement is valuable in the initial staging of ES/PNET. $\mathrm{BMAB}$ is considered to be the gold standard test for assessing bone marrow involvement, and its importance at initial staging is not well established. Moreover, BMAB is a painful procedure and has minimal but non-negligible adverse events such as hemorrhage and neurological complications [16]. Furthermore, the presence of bone marrow involvement does not affect the treatment strategy of patients with known distant metastases. Recent reports emphasized the finding suggesting that bone marrow involvements are unlikely to be observed if no metastases are detected on the imaging modalities $[13,17]$. Newman et al. retrospectively evaluated patients with ES/PNET who underwent both ${ }^{18} \mathrm{~F}$-FDG PET and BMAB of the iliac crest [13]. They demonstrated that 91 patients without distant metastases on ${ }^{18} \mathrm{~F}$-FDG PET had bone marrow involvement. Kopp reviewed patients with ES/ PNET that underwent evaluation for metastases using pulmonary CT, bone scan, and BMAB of the iliac crest [18]. They also showed that all the 103 patients with BMAB negative results were found to have no distant metastases on pulmonary $\mathrm{CT}$ and bone scan.

We retrospectively reviewed 26 patients with ES/PNET who underwent ${ }^{18} \mathrm{~F}$-FDG PET/CT and BMAB at initial evaluation; none of the 21 patients without distant metastases on ${ }^{18}$ F-FDG PET/CT had a positive BMAB result. Our result is concordant with that of previous literature. Therefore, this may support the suggestion to omit BMAB from routine work-up at the initial staging of ES/PNET patients without radiologic evidence of metastatic disease on ${ }^{18} \mathrm{~F}$ FDG PET/CT. Additionally, in the current study, bone marrow involvement was only detected in patients with bone metastases on ${ }^{18} \mathrm{~F}$-FDG PET/CT. Previous studies indicated that bone marrow involvement is frequently accompanied by bone metastasis, but that is not always the case [13,19]. Kopp et al. demonstrated that one patient among 13 BMABpositive patients had metastases, not to the bone but to the lung [18]. Thus, if distant metastasis is found on ${ }^{18} \mathrm{~F}-\mathrm{FDG}$ PET/CT, BMAB seems to be indicated, to detect the bone marrow involvement.

Secondly, our data demonstrated complete concordance in metastatic status between ${ }^{18} \mathrm{~F}$-FDG PET/CT and contrasted $\mathrm{CT}$ of the trunk in combination with MRI of the primary site. A recent meta-analysis reported that ${ }^{18} \mathrm{~F}$ - FDG PET/CT has high accuracy for detecting distant metastases of Ewing sarcoma, including the lung and bone metastases. However, almost all of the studies included in this meta-analysis were performed retrospectively [11]. Prospective trials should be carried out to assess the potential of ${ }^{18} \mathrm{~F}$ - FDG PET/CT and to optimize its use in the staging of ES/PNET.

Thirdly, we found primary tumor size and serum LDH level to be significantly higher in patients with bone marrow 
involvement as well as in patients with metastatic disease. The guidelines recommend measuring LDH level as a tumor marker since it usually correlates with tumor burden and has a prognostic value in ES/PNET $[9,10]$. Also, a meta-analysis indicated that patients with high serum levels of LDH have a poor prognosis compared to patients with normal serum $\mathrm{LDH}$ values [20]. Previous studies showed that tumor size of $8 \mathrm{~cm}$ or more is associated with poor survival $[3,21]$. Since both tumor size and LDH reflect the high tumor burden in the body and are well-recognized poor prognostic factors, we believe that it is natural that these factors correlate with bone marrow involvement and metastatic status.

There are several limitations to this study. Firstly, this is a single-center, retrospective study with small sample size. Therefore, we consider that the sample was too small to strictly determine the correlation between bone marrow involvement and all prognostic factors. Secondly, we used a morphological assessment to detect bone marrow involvement on BMAB specimen. There are more sensitive techniques for evaluating minimal bone marrow involvement, including flow cytometry and reverse transcriptase polymerase chain reaction (RT-PCR), though their usefulness in daily practice is unclear.

The strength of this study included the diverse patients' age groups (adolescents and adults). Also, this is the first study to compare the concordance in the metastatic status of ES/PNET between the results of BMAB and ${ }^{18} \mathrm{~F}-\mathrm{FDG}$ PET/CT.

\section{Conclusion}

The result of this study indicated that bone marrow involvement was confined to patients with metastases on ${ }^{18} \mathrm{~F}$-FDG PET/CT. Since the specificity of ${ }^{18} \mathrm{~F}$-FDG PET/CT for bone marrow involvement was $100 \%$ in our study, it may be a reasonable option to omit $\mathrm{BMAB}$ in patients who were suggested to have no metastatic sites on ${ }^{18} \mathrm{~F}$-FDG PET/CT. A prospective study is warranted to conclude on the value of ${ }^{18} \mathrm{~F}$-FDG PET/ $\mathrm{CT}$ in detecting bone marrow involvement and to determine the best initial workup of ES/PNET patients.

\section{Compliance with ethical standards}

Conflict of interest All authors declared no conflicts of interest.

Open Access This article is distributed under the terms of the Creative Commons Attribution 4.0 International License (http://creativeco mmons.org/licenses/by/4.0/), which permits unrestricted use, distribution, and reproduction in any medium, provided you give appropriate credit to the original author(s) and the source, provide a link to the Creative Commons license, and indicate if changes were made.

\section{References}

1. Carvajal R, Meyers P. Ewing's sarcoma and primitive neuroectodermal family of tumors. Hematol Oncol Clin North Am. 2005;19(3):501-25.

2. Sorensen PH, Lessnick SL, Lopez-Terrada D, Liu XF, Triche TJ, Denny CT. A second Ewing's sarcoma translocation, $t(21 ; 22)$, fuses the EWS gene to another ETS-family transcription factor, ERG. Nat Genet. 1994;6(2):146-51.

3. Cotterill SJ, Ahrens S, Paulussen M, Jurgens HF, Voute PA, Gadner $\mathrm{H}$, et al. Prognostic factors in Ewing's tumor of bone: analysis of 975 patients from the European Intergroup Cooperative Ewing's Sarcoma Study Group. J Clin Oncol. 2000;18(17):3108-14.

4. Gaspar N, Hawkins DS, Dirksen U, Lewis IJ, Ferrari S, Le Deley MC, et al. Ewing sarcoma: current management and future approaches through collaboration. J Clin Oncol. 2015;33(27):3036-46.

5. Kushner BH, Meyers PA. How effective is dose-intensive/myeloablative therapy against Ewing's sarcoma/primitive neuroectodermal tumor metastatic to bone or bone marrow? The Memorial Sloan-Kettering experience and a literature review. J Clin Oncol. 2001;19(3):870-80.

6. Kolb EA, Kushner BH, Gorlick R, Laverdiere C, Healey JH, LaQuaglia MP, et al. Long-term event-free survival after intensive chemotherapy for Ewing's family of tumors in children and young adults. J Clin Oncol. 2003;21(18):3423-30.

7. Oberlin O, Rey A, Desfachelles AS, Philip T, Plantaz D, Schmitt C, et al. Impact of high-dose busulfan plus melphalan as consolidation in metastatic Ewing tumors: a study by the Societe Francaise des Cancers de 1'Enfant. J Clin Oncol. 2006;24(24):3997-4002.

8. Felgenhauer J, Hawkins D, Pendergrass T, Lindsley K, Conrad EU 3rd, Miser JS. Very intensive, short-term chemotherapy for children and adolescents with metastatic sarcomas. Med Pediatr Oncol. 2000;34(1):29-38.

9. Casali PG, Bielack S, Abecassis N, Aro HT, Bauer S, Biagini $\mathrm{R}$, et al. Bone sarcomas: ESMO-PaedCan-EURACAN Clinical Practice Guidelines for diagnosis, treatment and follow-up. Ann Oncol. 2018;29(Supplement_4):iv79-95.

10. National Comprehensive Cancer Network. Bone Cancer (Version 1.2019). https://www.nccn.org/professionals/physician_gls/pdf/ bone.pdf.

11. Huang T, Li F, Yan Z, Ma Y, Xiong F, Cai X, et al. Effectiveness of $18 \mathrm{~F}-\mathrm{FDG}$ PET/CT in the diagnosis, staging and recurrence monitoring of Ewing sarcoma family of tumors: a meta-analysis of 23 studies. Medicine. 2018;97(48):e13457.

12. Wang J, Weiss LM, Chang KL, Slovak ML, Gaal K, Forman SJ, et al. Diagnostic utility of bilateral bone marrow examination. Cancer. 2002;94(5):1522-31.

13. Newman EN, Jones RL, Hawkins DS. An evaluation of [F-18]-fluorodeoxy-D-glucose positron emission tomography, bone scan, and bone marrow aspiration/biopsy as staging investigations in Ewing sarcoma. Pediatr Blood Cancer. 2013;60(7):1113-7.

14. Macpherson RE, Pratap S, Tyrrell H, Khonsari M, Wilson S, Gibbons M, et al. Retrospective audit of 957 consecutive 18F-FDG PET-CT scans compared to CT and MRI in 493 patients with different histological subtypes of bone and soft tissue sarcoma. Clin Sarcoma Res. 2018;8(1):9.

15. Oberlin O, Bayle C, Hartmann O, Terrier-Lacombe MJ, Lemerle $\mathrm{J}$. Incidence of bone marrow involvement in Ewing's sarcoma: value of extensive investigation of the bone marrow. Med Pediatr Oncol. 1995;24(6):343-6.

16. Bain BJ. Bone marrow biopsy morbidity: review of 2003. J Clin Pathol. 2005;58(4):406-8. 
17. Kasalak O, Glaudemans A, Overbosch J, Jutte PC, Kwee TC. Can FDG-PET/CT replace blind bone marrow biopsy of the posterior iliac crest in Ewing sarcoma? Skeletal Radiol. 2018;47(3):363-7.

18. Kopp LM, Hu C, Rozo B, White-Collins A, Huh WW, Yarborough $\mathrm{A}$, et al. Utility of bone marrow aspiration and biopsy in initial staging of Ewing sarcoma. Pediatr Blood Cancer. 2015;62(1):12-5.

19. Burdach S, van Kaick B, Laws HJ, Ahrens S, Haase R, Korholz $\mathrm{D}$, et al. Allogeneic and autologous stem-cell transplantation in advanced Ewing tumors. An update after long-term followup from two centers of the European Intergroup study EICESS. Stem-Cell Transplant Programs at Dusseldorf University Medical Center, Germany and St. Anna Kinderspital, Vienna, Austria. Ann Oncol. 2000;11(11):1451-62.
20. Li S, Yang Q, Wang H, Wang Z, Zuo D, Cai Z, et al. Prognostic significance of serum lactate dehydrogenase levels in Ewing's sarcoma: a meta-analysis. Mol Clin Oncol. 2016;5(6):832-8.

21. Fizazi K, Dohollou N, Blay JY, Guérin S, Le Cesne A, André F, et al. Ewing's family of tumors in adults: multivariate analysis of survival and long-term results of multimodality therapy in 182 patients. J Clin Oncol. 1998;16(12):3736-43.

Publisher's Note Springer Nature remains neutral with regard to jurisdictional claims in published maps and institutional affiliations. 